\title{
A Comparison of Methods for Recovering Intra-voxel White Matter Fiber Architecture from Clinical Diffusion Imaging Scans
}

\author{
Alonso Ramirez-Manzanares, Philip A. Cook, and James C. Gee \\ Penn Image Computing and Science Laboratory (PICSL), Department of Radiology, \\ University of Pennsylvania, Philadelphia, PA, USA \\ \{alram, cookpa, gee\}@mail.med.upenn.edu
}

\begin{abstract}
Diffusion tensor magnetic resonance imaging is widely used to study the structure of the fiber pathways of brain white matter. However, the diffusion tensor cannot capture complex intra-voxel fiber architecture such as fiber crossings. Consequently, a number of methods have been proposed to recover intra-voxel fiber bundle orientations from high angular-resolution diffusion imaging scans, which are optimized to resolve fiber crossings. In this work we study how multi-tensor, spherical deconvolution, analytical QBall and diffusion basis function methods perform under clinical scanning conditions. Our experiments indicate that it is feasible to apply some of these methods in clinical data sets.
\end{abstract}

Keywords: DW-MRI, crossing fibers, HARDI, Multi-DT, QSpace.

\section{Introduction}

The most widely-used approach to study water diffusion in the human brain is diffusion tensor imaging (DTI) 11, where the diffusion tensor's (DT) main eigenvector corresponds to the axis of maximum diffusion. In white matter fiber tracts, the main eigenvector is aligned with the local average orientation of the fibers, making it possible to study patterns of brain connectivity in-vivo.

The chief limitation of DTI is that the DT is constrained to represent only one maximum diffusion orientation and thus it is inadequate in voxels where two or more fiber bundles cross, split or "kiss". This represents a significant problem for diffusion tractography, where we rely on local fiber-orientation estimates to reconstruct fiber pathways. According to [2], as many as one third of white-matter voxels contain more than one fiber bundle orientation. A number of methods have been developed to resolve heterogeneous intra-voxel fiber structures, 3] gives a review. In this study we focus on approaches that use data acquired at a fixed "b-value" with independent gradient directions, which is the type of data usually acquired for DTI. DTI scans use b-values of approximately $1000 \mathrm{~s} / \mathrm{mm}^{2}$ for optimal performance [4, and can be computed with a minimum of six gradient orientations. High angular resolution diffusion imaging (HARDI) scans typically use a relatively large set of gradient directions and higher b-values, to develop the contrast for multi-fiber reconstruction.

D. Metaxas et al. (Eds.): MICCAI 2008, Part I, LNCS 5241, pp. 305-312, 2008.

(C) Springer-Verlag Berlin Heidelberg 2008 
Recent improvements in the speed of image acquisition, combined with demand for high-quality DT data, have led to an increased adoption of DTI acquisition protocols using 30 or more gradient directions, which opens the possibility of applying HARDI methods in clinical scans. It is therefore useful to investigate how the HARDI methods perform under contemporary DTI scanning protocols. Even though most of reported methods provide some experimental validation, to the best of our knowledge there is not a reported comparison for several methods under the same conditions using a clinically realistic DTI acquisition protocol. In this paper, we compare five previously published HARDI methods under the same simulated acquisition settings for synthetic as well as for human DW-MRI data. The methods are explained in the following section.

\subsection{Methods for Intra-voxel Fiber Estimation}

First, we introduce the basic models for the normalized DW signal $A(\mathbf{q})$ given the diffusion wavenumber $\mathbf{q}$ :

$$
\begin{aligned}
& A(\mathbf{q})=\int_{R^{3}} p(\mathbf{x}) \cos \left(\mathbf{q}^{T} \mathbf{x}\right) d \mathbf{x} \\
& A(\mathbf{q})=\sum_{i=1} \alpha_{i} \exp \left(-\mathbf{q}^{T} \mathbf{D}_{i} \mathbf{q} \tau\right) \\
& A(\mathbf{q})=\int_{R^{3}} A\left(\mathbf{q} ; \mathbf{x}_{0}\right) f(\mathbf{x}) d \mathbf{x} .
\end{aligned}
$$

Model (1) involves the particle displacement Probability Density Function (PDF) $p$, (2) is the Gaussian Mixture Model (GMM) and (3) associates the Fiber Orientation Distribution (FOD) $f$ estimated by Spherical Deconvolution (SD). DT's are denoted by $\mathbf{D}_{j}$ with contribution $\alpha_{i} \in[0,1], \tau$ is the effective diffusion time and $A\left(\mathbf{q} ; \mathbf{x}_{0}\right)$ is the DW signal for a fiber along orientation $\mathbf{x}_{0}$, see 3 .

For our comparison study we select a set of methods from the literature: Multi-DT (MDT) [5], analytical QBall (AQBl) [6], Maximum Entropy Spherical Deconvolution (MESD) 7], Non-negativity Constrained Super-Resolved Spherical Deconvolution (SCSD) [8], and Diffusion Basis Functions (DBF) 9]. Next we briefly describe these methods (see [10] for a study of similarities among them).

1. MDT [5]. This method assumes the GMM (2). The fitting procedure requires non-linear optimization. In [5] the DT's eigenvalues were fixed and the GMM was fitted by a multi-start gradient descent algorithm. We optimize the full diffusion tensors subject to non-negativity of the eigenvalues, using a Levenberg-Marquardt algorithm [11. A problem for this method is the need for model selection as a pre-processing step.

2. AQBl [6]. By using the Funk transform, a projection of $p$ along the orientation $\mathbf{x}$ is proportional to the integral of $A(\mathbf{q})$ over the circle perpendicular to $\mathbf{x}$. We use the novel analytical reconstruction approach which introduces a regularization term based on the Laplace-Beltrami operator. This modification improves fiber orientation detection. The non-parametric nature allows 
one to recover an arbitrary number of fiber orientations but makes the estimation noise sensitive.

3. MESD [7]. This is a generalization of the Persistent Angular Structure (PAS) method. The PAS function is parameterized by a maximum-entropy model. The PDF estimation is achieved by evaluating an integral only for a fixed radius sphere in $\mathbf{q}$ space. This representation allows one to recover high quality solutions of the angular structure of $p$, although the required non-linear optimization is computationally expensive.

4. SCSD [8]. This proposal extends previous proposals on SD which deconvolve the FOD $f$ from model (3). This work tackles the ill-conditioned problem of classical SD approaches by constraining the non-negativity of the FOD components, which allows super-resolution: the FOD can be estimated with more parameters than measured signals.

5. DBF [9]. A discrete version of the GMM is proposed by fixing a tensor basis consisting of anisotropic diffusion tensors with principal directions isotropically distributed on the sphere. A dictionary of DBF is computed (one signal per basis tensor), so that $A(\mathbf{q})$ is explained as a non-negative linear combination of the DBF. The fitting procedure is based on a Basis Pursuit optimization framework which searches for the smallest possible number of DBF to represent the signal.

The rest of the paper is organized as follows. We explain the comparison framework in section 2 The results are presented in section 3 and finally we discuss the experiments and present our conclusions in Section 4.

\section{Experimental Methods}

To implement the various methods, we use: a) the code provided by the authors for methods MESD, SCSD, AQBl and DBF, and b) open-source implementations for method MDT in the Camino toolkit [11. Also, we use the proposed parameters supplied with the software as described below. For AQBl we use harmonic order $l=8$ and regularization amount $\lambda_{L B}=0.006$; no FOD sharpening is applied. For the MESD method we use a PAS filter with radius 1.4. For SCSD we use spherical harmonic order $l_{\max }=10, \lambda=1, \tau=10 \%$. For DBF method we use the reported 129 basis orientations. Peak detection was performed for AQBl, MESD and SCSD by using the Camino toolkit [11. Following [8], peaks smaller than $20 \%$ of the magnitude of the largest peak were eliminated. Correspondingly, for DBF and MDT we eliminated tensors with coefficients $\alpha_{i}$ less than $20 \%$ of the largest coefficient. We provided to SCSD and DBF the same profile signal for a single fiber bundle $A\left(\mathbf{q} ; \mathbf{x}_{0}\right)$, which is computed using the average DT in voxels thought to contain a single fiber bundle. In the brain data, we fitted the DT to the data set, then we calculate average eigenvalues from tensors with linear coefficient larger than both spheric and planar coefficients, which are defined in 12 . In the synthetic data, we generated synthetic noisy measurements from voxels containing a single diffusion tensor. 
We use data from three sources in the experiments:

1. Synthetic data - free diffusion model. The DW-MRI signal was synthesized from the GMM (2). The DT principal eigenvalue was set to $1 \times 10^{-3}$ $\mathrm{mm}^{2} / \mathrm{s}$ and the second and third eigenvalues were $2.22 \times 10^{-4} \mathrm{~mm}^{2} / \mathrm{s}$, so that the Fractional Anisotropy (FA) [1] is equal to 0.74 and the diffusion ratio (longitudinal/transversal) is equal to 4.5. The above values were taken from a sample of tensors observed in the brain data from a healthy volunteer (see below). The number of tensors in each voxel of synthetic data is either two or three and the crossing angle is varied between 30 and 90 degrees. A random rotation was applied to all tensors in each voxel before the data was generated, to simulate fiber bundles at different orientations.

2. Synthetic data - restricted diffusion model. The MDT and the DBF methods both use the GMM, so they have an advantage when data is synthesized from a GMM. We therefore perform one experiment with data generated from a Monte-Carlo (MC) simulation of diffusion within and around impermeable cylinders. For the simulation, $10^{5}$ simulated water molecules were evenly distributed on a substrate consisting of a regular grid of impermeable, hollow cylinders of radius $5 \times 10^{-6} \mathrm{~m}$, separated by $13 \times 10^{-6}$ m. The simulated MR acquisition was designed to be as similar as possible to the human brain acquisition (below): nine measurements at $b=0$ and sixty at $b=1000 \mathrm{~s} / \mathrm{mm}^{2}$, diffusion time $\Delta=0.035 \mathrm{~s}$ and pulse width $\delta=0.017 \mathrm{~s}$. The algorithm for simulating the diffusion and the MRI acquisition is explained in 13 and implemented in the Camino toolkit [1]. When the diffusion tensor is fitted to this data, the FA is close to 0.74 , as is used in the GMM experiments. To generate data from a fiber crossing, we rotate half of the cylinders by 90 degrees and repeat the simulation.

3. Human brain data. A single healthy volunteer was scanned on a Siemens Trio 3T scanner. The DWI acquisition parameters were as follows: singleshot echo-planar imaging, nine images for $\mathrm{b}=0 \mathrm{~s} / \mathrm{mm}^{2}, 60 \mathrm{DW}$ images with unique, isotropically distributed orientations $\left(b=1000 \mathrm{~s} / \mathrm{mm}^{2}\right), T R=6700$ $\mathrm{ms}, \mathrm{TE}=85 \mathrm{~ms}, 90^{\circ} \mathrm{flip}$ angle, voxel dimensions equal to $2 \times 2 \times 2 \mathrm{~mm}^{3}$. We compute the fiber orientations using each method, first using all 60 of the diffusion weighted measurements, and then with subsets of various sizes. The purpose of this experiment is to report the method's stability if fewer than 60 measurements were acquired. In order to preserve the isotropic distribution of directions, we compute evenly-distributed subsets using the method presented in [14].

\section{Results}

The top part of Table 1 presents results for the synthetic signal generated with free diffusion model. The results show the following configurations of the GMM: a) a two fiber crossing with random crossing angle $60^{\circ}>\gamma_{i}<90^{\circ}$, denoted as $2 \approx \widetilde{90}$, b) a three fiber crossing with random crossing angle $60^{\circ}>\gamma_{i}<90^{\circ}$, denoted as $3 \widetilde{90}$, and c) a two fiber crossing with crossing angle $\gamma_{i}$ denoted as $2_{\gamma_{i}}$. Sixty 
Table 1. Numerical results for comparison with synthetic signals (free diffusion and cylinder restricted models). We show in bold the best results per category.

Free Diffusion

\begin{tabular}{|c|c|c|c|c|c|c|c|c|c|c|c|c|c|c|c|c|}
\hline & \multicolumn{15}{|c|}{ Free Diffusior } \\
\hline & & \multicolumn{5}{|c|}{$\mathrm{SNR}=10$} & \multicolumn{5}{|c|}{$\mathrm{SNR}=20$} & \multicolumn{5}{|c|}{$\mathrm{SNR}=30$} \\
\hline & & $n_{-}$ & $\bar{n}_{+}$ & $\bar{\epsilon}_{\theta}$ & $\sigma_{\epsilon_{\theta}}$ & $\bar{\epsilon}_{\alpha}$ & $\bar{n}_{-}$ & $\bar{n}_{+}$ & $\bar{\epsilon}_{\theta}$ & $\sigma_{\epsilon_{\theta}}$ & $\bar{\epsilon}_{\alpha}$ & $\bar{n}_{-}$ & $\bar{n}_{+}$ & $\bar{\epsilon}_{\theta}$ & $\sigma_{\epsilon_{\theta}}$ & $\bar{\epsilon}_{\alpha}$ \\
\hline & $2 \widetilde{90}$ & 0.2 & & $\overline{18.9}$ & 12.2 & 0.20 & $\overline{0.1}$ & & 11.7 & 10.1 & 0.15 & || 0.0 & & 7.6 & $\overline{6.3}$ & 0.12 \\
\hline M & $3 \approx \widetilde{90}$ & 0.3 & & 27.7 & 14.8 & 0.18 & & & 25.5 & 14.8 & 0.25 & 0.8 & & 24.1 & 13.8 & $2^{2}$ \\
\hline $\mathrm{D}$ & 230 & 0.5 & & 18.2 & 15.6 & 0.28 & 0 & & & 11.7 & 0.31 & || 0.6 & & 13.7 & 10.7 & 0.34 \\
\hline$\Gamma$ & 240 & 4 & & 18.6 & 13.7 & 0.28 & & & 5.9 & 10.0 & 0.30 & 0.5 & & 4.2 & 9.8 & 0.29 \\
\hline & 250 & 0.3 & & 20.3 & 12.1 & 0.26 & & & .5 & 10.5 & 26 & 0.3 & & 3.1 & 7.9 & J. 2 \\
\hline & 260 & 0.3 & & 19.2 & 11.1 & 0.23 & 0.2 & & 14.0 & 8.2 & 0.22 & 0.0 & & 10.6 & 7.3 & 0.18 \\
\hline & $2 \widetilde{90}$ & 0.6 & 0.0 & 19.8 & 11.4 & 0.31 & $\overline{7}$ & 0.0 & 20.3 & 11.0 & 0.37 & 0.8 & 0.0 & $\overline{20.6}$ & $\overline{\overline{10.0}}$ & $\overline{\overline{0.40}}$ \\
\hline$A$ & $3 \approx$ & & 0 & 28.7 & 5.4 & 0.25 & & 0.0 & 28.9 & 12.8 & 0.40 & .5 & 0.0 & 29.2 & 11.8 & 0.4 \\
\hline Q & 230 & & & .5 & 3.7 & 0.50 & & 0.0 & 12.3 & 2.6 & 0.50 & .0 & 0.0 & 12.9 & 1. & .00 \\
\hline B & 240 & 1.0 & 0.0 & 9 & 5.2 & 0.50 & 0 & 0.0 & 16.8 & 3.2 & 0.50 & 1.0 & 0.0 & 17.5 & 2.3 & 0.50 \\
\hline 1 & $2_{50}$ & م & 0.0 & 18.5 & 6.3 & 0.50 & & 0.0 & 21.0 & 3.8 & 0.50 & 1.0 & 0.0 & 22.0 & 2.8 & 0.5 \\
\hline & $2_{60}$ & 0.9 & 0.0 & 22.0 & 9.3 & 0.45 & 1.0 & 0.0 & 24.0 & 5.5 & 0.50 & 1.0 & 0.0 & 25.5 & 4.1 & 0.5 \\
\hline & $2 \approx \widetilde{90}$ & 0 & 7 & 1.7 & $\overline{2.7}$ & 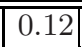 & 0.0 & 0.4 & 14.5 & 10.5 & 0.10 & 0.0 & 0.1 & 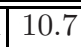 & 8. & 0.0 \\
\hline M & $3 \approx$ & 0.0 & 0.0 & 26.1 & 14.1 & 0.08 & 0.0 & 0.0 & 20.1 & 12.4 & 0.08 & 0.1 & 0.0 & 16.5 & \begin{tabular}{|l}
11.7 \\
\end{tabular} & 0.0 \\
\hline $\mathrm{E}$ & 230 & .3 & .3 & 24.1 & 21.1 & 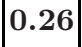 & ( 8 & 0.1 & 14.7 & 17.5 & 0. & 0.9 & 0.0 & 12.2 & 11. & 0 . \\
\hline S & 240 & 2 & 5 & 23.6 & 7.0 & 1 & 4 & 2 & 9.6 & 17.0 & 0.26 & 0.8 & 0.0 & 14.9 & 12.1 & 0.38 \\
\hline $\mathrm{D}$ & $2_{50}$ & 0.1 & 5 & 23.9 & 5.6 & 7 & 2 & 0.2 & 19.5 & 13.1 & 0.21 & 0.3 & 0.0 & 16.3 & 12.5 & 0.2 \\
\hline & 260 & 0.0 & 0.6 & 22.5 & 3.5 & 4 & \pm & .3 & 17.8 & 11.9 & 0.15 & 0.1 & 0.1 & 13.3 & 9.1 & 0.1 \\
\hline & $2 \widetilde{90}$ & $\overline{.1}$ & 0.0 & 19.1 & 13.8 & 0.11 & & 0.0 & $\overline{12.6}$ & 11.1 & 0.08 & \begin{tabular}{|c|}
0.1 \\
\end{tabular} & 0.0 & 8.9 & 7.8 & 0.07 \\
\hline S & $3 \approx \widetilde{9}$ & 0.2 & 0.0 & 26.2 & 13.6 & 0.10 & .2 & 0.0 & 22.2 & 13.3 & 0.08 & 0.1 & 0.0 & 17.0 & 11.5 & 0.07 \\
\hline $\mathrm{Cl}$ & $2_{30}$ & 1.0 & 0.0 & 12.7 & 6.6 & 0.47 & 0 & 0.0 & 13.3 & 1.5 & 0.49 & 1.0 & 0.0 & 13.8 & 0.9 & 0.50 \\
\hline 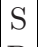 & $2_{40}$ & & 0.0 & 16.0 & 11.4 & 0.45 & & 0.0 & 15.4 & 4.2 & 0.47 & 1.0 & 0.0 & 16.4 & 6.9 & 0.4 \\
\hline $\mathrm{D}$ & $2_{50}$ & 7 & 0.0 & 19.1 & 12.1 & 0.38 & 8 & 0.0 & 16.5 & 9.6 & 0.43 & 0.8 & 0.0 & 15.0 & 9.3 & 0.45 \\
\hline & $2_{60}$ & 0.2 & 0.0 & 20.0 & 12.5 & 0.21 & 0.2 & 0.0 & 15.7 & 11.5 & 0.20 & 0.2 & 0.0 & 12.1 & 8.4 & 0.18 \\
\hline & $2 \widetilde{90}$ & & 5 & 17.9 & 12.2 & 11 & $\overline{0.0}$ & 0.4 & 11.5 & .0 & $\overline{5}$ & 0.0 & 0.4 & 6.8 & 5. & 0.07 \\
\hline $\mathrm{D}$ & $3 \approx \widetilde{90}$ & 0.0 & 0.0 & 26.7 & 14.7 & 0.08 & 0.0 & 0.0 & 21.5 & 14.9 & 0.06 & || 0.0 & 0.0 & 16.4 & 12.8 & 0.06 \\
\hline 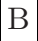 & $2_{30}$ & 0.6 & 0.1 & 12.6 & 7.0 & 0.31 & & 0.0 & 11.7 & 4.1 & 0.35 & 0.7 & 0.0 & 11.5 & 3.9 & 0.36 \\
\hline $\mathrm{F}$ & 240 & 0.4 & 0.1 & 16.0 & 10.9 & 0.26 & 0.3 & 0.2 & 13.5 & 7.1 & 0.24 & 0.2 & 0.1 & 12.0 & 6.7 & 0.23 \\
\hline & $2_{50}$ & 0.2 & 0.2 & 17.4 & 10.6 & 0.20 & 0.1 & 0.2 & 13 & 9.0 & 0.18 & 0.1 & 0.3 & 10.7 & 6.2 & 0.16 \\
\hline & $2_{60}$ & 0.0 & 0.3 & 18.0 & 10.9 & 0.15 & 0.0 & 0.3 & 12.6 & 9.0 & 0.13 & 0.0 & 0.3 & 8.8 & 6.2 & 0.10 \\
\hline
\end{tabular}

MC Cylinder restricted diffusion, 2 Fibers $90^{\circ}$

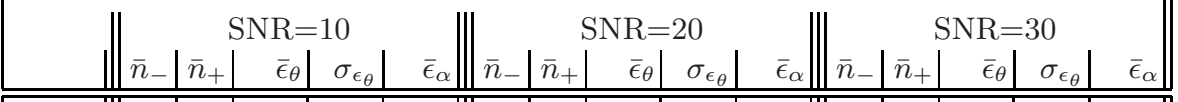
\begin{tabular}{|l||l|l|r|r|r||l|l|r|r|r||l|l|r|r|r||}
\hline MDT & $\mathbf{0 . 0}$ & & 9.1 & 5.4 & 0.10 & $\mathbf{0 . 0}$ & & 4.1 & 2.2 & 0.06 & $\mathbf{0 . 0}$ & & 2.7 & $\mathbf{1 . 4}$ & 0.04 \\
\hline \hline
\end{tabular}

\begin{tabular}{|c|c|c|c|c|c|c|c|c|c|c|c|c|}
\hline$\overline{\mathrm{AQBl}}$ & \begin{tabular}{l|l}
0.1 & $\mathbf{0 . 0}$ \\
\end{tabular} & 12.2 & 7.4 & 0.08 & \begin{tabular}{|l|l|}
0.0 & 0.0 \\
\end{tabular} & 6.6 & \begin{tabular}{l|l}
4.2 \\
\end{tabular} & "0.03 & \begin{tabular}{|l|l|}
0.0 & 0.0 \\
\end{tabular} & 4.4 & 2.7 & 0.03 \\
\hline$\overline{\mathrm{MESD}}$ & \begin{tabular}{l|l|}
0.0 & 0.0 \\
\end{tabular} & 8.6 & 4.5 & 0.05 & \begin{tabular}{|l|l|}
0.0 & 0.0 \\
\end{tabular} & 4.2 & 2.0 & 0.02 & \begin{tabular}{|l|l|}
0.0 & 0.0 \\
\end{tabular} & 2.8 & 1.4 & .02 \\
\hline$\overline{\mathrm{SCSD}}$ & \begin{tabular}{||l|l|}
0.0 & 0.0 \\
\end{tabular} & 11.1 & 5.6 & 0.10 & \begin{tabular}{||l|l|}
0.0 & 0.0 \\
\end{tabular} & 6.2 & 3.5 & 0.13 & \begin{tabular}{|l|l|}
0.0 & 0.0 \\
\end{tabular} & 4.4 & 2.5 & $\overline{0.14}$ \\
\hline$\overline{\mathrm{DBF}}$ & \begin{tabular}{||l|l|}
$\mathbf{0 . 0}$ & 0.4 \\
\end{tabular} & 8.2 & 5.8 & 0.07 & \begin{tabular}{|l|l|}
$\mathbf{0 . 0}$ & 0.1 \\
\end{tabular} & 3.5 & 2.5 & 0.03 & \begin{tabular}{|l|l|}
0.0 & 0.0 \\
\end{tabular} & 2.1 & 1.6 & .02 \\
\hline
\end{tabular}


Table 2. Comparison for human brain data, entries show how the solution changes as

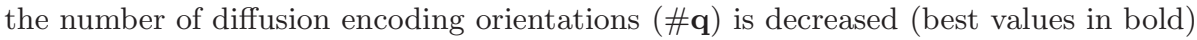

\begin{tabular}{|c|c|c|c|c|c|c|c|c|c|c|c|}
\hline$\#$ & \multicolumn{3}{|c|}{ MESD } & \multicolumn{4}{|c|}{ SCSD } & \multicolumn{4}{|c|}{$\mathrm{DBF}$} \\
\hline$q$ & \begin{tabular}{l|l|}
$\bar{n}_{-}$ & $\bar{n}_{+}$ \\
\end{tabular} & $\bar{\epsilon}_{\theta}$ & $\sigma_{\epsilon_{\theta}}$ & \begin{tabular}{l|l}
$\bar{n}_{-}$ & $\bar{n}_{+}$ \\
\end{tabular} & $\bar{\epsilon}_{\theta}$ & $\sigma_{\epsilon_{\theta}}$ & $\bar{\epsilon}_{\alpha}$ & $\mid \bar{n}_{-} \bar{n}_{+}$ & $\bar{\epsilon}_{\theta}$ & $\sigma_{\epsilon_{\theta}}$ & $\bar{\epsilon}_{\alpha}$ \\
\hline 50 & \begin{tabular}{|l|l|}
0.1 & 0.1 \\
\end{tabular} & 6.9 & \begin{tabular}{|l|l|}
9.7 & 0.06 \\
\end{tabular} & \begin{tabular}{||l|l|}
0.1 & 0.1 \\
\end{tabular} & 10.0 & 12.9 & 0.07 & \begin{tabular}{||l|l|}
0.2 & $\mathbf{0 . 1}$ \\
\end{tabular} & 8.2 & 14.1 & 0.07 \\
\hline 40 & \begin{tabular}{|l|l|}
0.1 & 0.1 \\
\end{tabular} & 9.8 & \begin{tabular}{|l|l|}
11.7 & 0.08 \\
\end{tabular} & \begin{tabular}{||l|l|}
0.1 & 0.1 \\
\end{tabular} & 16.0 & 15.0 & 0.09 & \begin{tabular}{||l|l|}
0.2 & $\mathbf{0 . 1}$ \\
\end{tabular} & 10.8 & 15.2 & 0.09 \\
\hline 30 & \begin{tabular}{|l|l|}
0.1 & 0.1 \\
\end{tabular} & 2.9 & 13.1 & \begin{tabular}{|l|l|}
$\mathbf{0 . 1}$ & 0.2 \\
\end{tabular} & 16.0 & 14.3 & 0.10 & \begin{tabular}{||l|l|}
0.2 & 0.2 \\
\end{tabular} & $\overline{13.5}$ & $\overline{15.9}$ & 0.11 \\
\hline
\end{tabular}

voxels of synthetic data are generated for each configuration. Rician noise was added to each measurement to produce the desired signal to noise ratio (SNR) in the $b=0$ images. For each voxel, given the ground-truth and a set of recovered orientations, we first compute the number of axon bundles not recovered, $n_{-}$; or the number of extra bundles $n_{+}$found by the reconstruction - if the number of fiber bundles matches the true value, both of these quantities are zero. To compute the orientation error, we match the orientations returned from the methods to the ground truth, pairing directions such that the error is minimized. Thus the angle error $\epsilon_{\theta}$ and the absolute difference of the size compartments $\epsilon_{\alpha}$ are computed for each ground-truth orientation. We compute the mean value for the errors and the standard deviation for the angular error $\left(\sigma_{\epsilon_{\theta}}\right)$. All of the error metrics are defined such that the smallest number (shown in bold font) is the best result. The comparison of the methods in the MC simulation experiment is shown on the bottom part of Table 1. For these experiments we generate 60 voxels of data by adding noise to the data synthesized from the single-fiber and the crossing-fiber simulations.

For human brain data we show a qualitative comparison in Figure 1 for the three most successful methods: MESD, SCSD and DBF. For the visualization of MESD and SCSD, we use the same diffusion tensors as in the DBF solution, aligned to the MESD and SCSD peaks and scaled by the strength of each peak. For the DBF visualization, tensors are scaled by the contribution of each basis

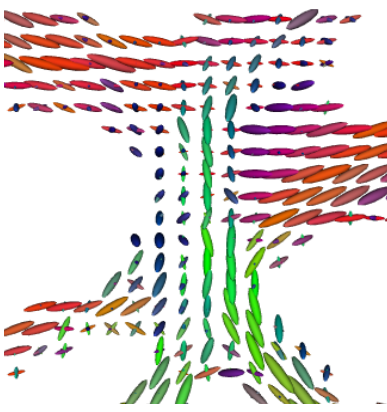

(a) MESD

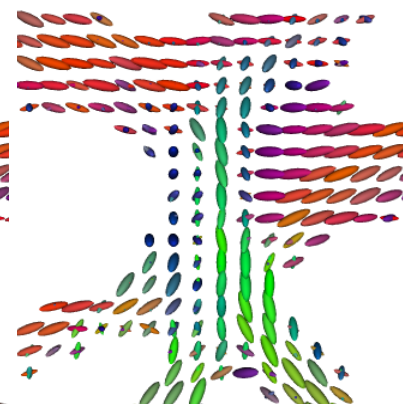

(b) SCSD

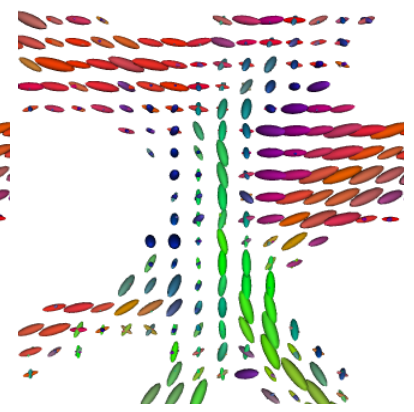

(c) $\mathrm{DBF}$

Fig. 1. Results for different methods in brain DW-MRI, the image shows the intersection of posterior corona radiata and tapetum-splenium of corpus callosum 
function to the solution. We note that there is not a significant difference in the solutions: the mean difference error among them is $\bar{\epsilon}_{\theta} \approx 8.5^{\circ}, \bar{n}_{-} \approx 0.21$ and $\bar{n}_{+} \approx 0.21$ for 936 voxels. The performance comparison in human data tests the consistency of the methods when there is less available DW-MRI data. The "ground truth" is the fiber orientations calculated by using all of the data $(\# \mathbf{q}=60)$ and report in Table 2 how the results differ for each as we diminish the number of diffusion encoding orientations (\# $\mathbf{q} \in[50,40,30])$. The MESD method performs most consistently with less DW-MRI data.

\section{Discussion and Conclusions}

For the synthetic data results in Table 1. the MDT method produces good results compared to some other methods, though here we indicate a priori the model to fit ( 2 or $3 \mathrm{DT}$ ). In real data we would have to use a separate model selection procedure, however, it appears that this method would perform well given accurate model selection.

The synthetic results also suggest that AQBl, at least in the implementation used here, cannot be successfully applied to scans acquired under our $b=1000$ $\mathrm{s} / \mathrm{mm}^{2} / 60$-direction DTI protocol. It presents high $\bar{n}_{-}$values which indicate a systematic underestimation of the number of fiber bundles. For the 30 degree crossing angle all methods are detecting only one fiber in most cases, according to the high $\bar{n}_{-}$values. The SCSD method also presents the smallest overestimation of the number of fiber orientations, but it is also more likely to underestimate the number of fiber bundles than MESD and DBF. The DBF method produces the smallest angular error, and performs similarly to MESD in estimating the number of fiber orientations. The relative performance of the methods is unchanged when synthesizing data from the GMM or from a MC diffusion simulation.

For the human brian data results in Table 2 and Figure1, the MESD method is most robust when the number of diffusion encoding orientations is reduced, followed by DBF and SCSD respectively. The results for 60 diffusion encoding orientations (in Figure 1) are very similar for MESD, SCSD and DBF methods.

Regarding computation effort, MESD is the most expensive (with an average of $57 \mathrm{~s}$ per voxel, Java implementation), followed by SCSD (1.36 s per voxel, Matlab implementation) and $\operatorname{DBF}(0.16 \mathrm{~s}$ per voxel, Matlab implementation) respectively (on an AMD Opteron $2.4 \mathrm{GHz} \mathrm{CPU}$ ). This study shows that it is possible to recover competitive estimations with low computational burden (i.e. by using SCSD or DBF).

The main contribution of this paper is the comparison of how multi-fiber methods perform in a realistic DTI data set. The results in synthetic data suggest that a small improvement in accuracy can be gained by using DBF, though the difference in angular error is fairly small (approximately 2 degrees). The DBF calculation is significantly faster than MESD and comparable to SCSD. MESD performs best in human brain data with fewer diffusion gradient directions, however this comes at the expense of much longer computation time. 
Acknowledgments. We thank Maxime Descoteaux, Rachid Deriche and Donald Tournier for the provision of code, we also thank Kiran Seunarine and Matt Hall for help with the Camino toolkit. The authors gratefully acknowledge the NIH for support of this work through grants HD042974, HD046159, MH068066 and NS045839. This research was supported in part by CONACYT Mexico by a postdoctorate scholarship to A. Ramirez-Manzanares.

\section{References}

1. Basser, P.J., Pierpaoli, C.: Microstructural and physiological features of tissues elucidated by quantitative DT-MRI. J. Magn. Reson. B 111, 209-219 (1996)

2. Behrens, T.E.J., Berga, H.J., Jbabdi, S., Rushworth, M.F.S., Woolrich, M.W.: Probabilistic diffusion tractography with multiple fibre orientations: What can we gain? NeuroImage 34(1), 144-155 (2007)

3. Alexander, D.C.: Multiple-fibre reconstruction algorithms for diffusion MRI. Annals of the New York Academy of Sciences 1046, 113-133 (2005)

4. Alexander, D.C., Barker, G.J.: Optimal imaging parameters for fiber-orientation estimation in diffusion MRI. NeuroImage 27, 357-367 (2005)

5. Tuch, D.S., Reese, T.G., Wiegell, M.R., Makris, N., Belliveau, J.W., Wedeen, V.J.: High angular resolution diffusion imaging reveals intravoxel white matter fiber heterogeneity. Magn. Reson. Med. 48(4), 577-582 (2002)

6. Descoteaux, M., Angelino, E., Fitzgibbons, S., Deriche, R.: Regularized, fast and robust analytical Q-ball imaging. Magn. Reson. Med. 58(3), 497-510 (2007)

7. Alexander, D.C.: Maximum entropy spherical deconvolution for diffusion MRI. In: Proc. Inf. Processing Med. Imaging, Glenwood Springs, CO, USA, pp. 76-87 (2005)

8. Tournier, J.D., Calamante, F., Connelly, A.: Robust determination of the fibre orientation distribution in diffusion MRI: Non-negativity constrained super-resolved spherical deconvolution. NeuroImage 35(4), 1459-1472 (2007)

9. Ramirez-Manzanares, A., Rivera, M., Vemuri, B.C., Carney, P., Mareci, T.: Diffusion basis functions decomposition for estimating white matter intravoxel fiber geometry. IEEE Trans. Med. Imaging 26(8), 1091-1102 (2007)

10. Jian, B., Vemuri, B.C.: A unified computational framework for deconvolution to reconstruct multiple fibers from DW-MRI. IEEE Trans. Med. Imaging 26(11), 14641471 (2007)

11. Cook, P.A., Bai, Y., Nedjati-Gilani, S., Seunarine, K.K., Hall, M.G., Parker, G.J., Alexander, D.C.: Camino: Open-source diffusion-MRI reconstruction and processing. In: Proc. 14th Scientific Meeting of the ISMRM, Seattle, WA, USA, p. 2759 (2006)

12. Westin, C.F., Peled, S., Gudbjartsson, H., Kikinis, R., Jolesz, F.A.: Geometrical diffusion measures for MRI from tensor basis analysis. In: Proc. 5th Scientific Meeting of the ISMRM, Vancouver, Canada, p. 1742 (1997)

13. Hall, M.G., Alexander, D.C.: Finite pulse width improve fibre orientation estimates in diffusion tensor MRI. In: Proc. 14th Scientific Meeting of the ISMRM, Seattle, WA, USA, p. 1076 (2006)

14. Cook, P.A., Symms, M., Boulby, P.A., Alexander, D.C.: Optimal acquisition orders of diffusion-weighted MRI measurements. J. Magn. Reson. Imaging 25(5), 1051$1058(2007)$ 